\title{
Spectral Properties of Holstein and Breathing Polarons
}

\author{
C. Slezak ${ }^{1}$, A. Macridin ${ }^{1}$, G. A. Sawatzky ${ }^{2}$, M. Jarrell ${ }^{1}$ and T.A. Maier ${ }^{3}$ \\ ${ }^{1}$ University of Cincinnati, Cincinnati, Ohio, 45221, USA \\ ${ }^{2}$ University of British Columbia, 6224 Agricultural Road, Vancouver, BC V6T 1Z1, Canada \\ ${ }^{3}$ Oak Ridge National Laboratory, Oak Ridge, Tennessee, 37831, USA
}

(Dated: October 8, 2018)

\begin{abstract}
We calculate the spectral properties of the one-dimensional Holstein and breathing polarons using the self-consistent Born approximation. The Holstein model electron-phonon coupling is momentum independent while the breathing coupling increases monotonically with the phonon momentum. We find that for a linear or tight binding electron dispersion: i) for the same value of the dimensionless coupling the quasiparticle renormalization at small momentum in the breathing polaron is much smaller, ii) the quasiparticle renormalization at small momentum in the breathing polaron increases with phonon frequency unlike in the Holstein model where it decreases, iii) in the Holstein model the quasiparticle dispersion displays a kink and a small gap at an excitation energy equal to the phonon frequency $\omega_{0}$ while in the breathing model it displays two gaps, one at excitation energy $\omega_{0}$ and another one at $2 \omega_{0}$. These differences have two reasons: first, the momentum of the relevant scattered phonons increases with increasing polaron momentum and second, the breathing bare coupling is an increasing function of the phonon momentum. These result in an effective electron-phonon coupling for the breathing model which is an increasing function of the total polaron momentum, such that the small momentum polaron is in the weak coupling regime while the large momentum one is in the strong coupling regime. However the first reason does not hold if the free electron dispersion has low energy states separated by large momentum, as in a higher dimensional system for example, in which situation the difference between the two models becomes less significant.
\end{abstract}

\section{INTRODUCTION}

Interest in the spectral properties of strongly coupled electron-phonon systems has increased due to the discovery of a kink in the quasiparticle dispersion ${ }^{1,2,3,4}$ measured by angle-resolved photoemission (ARPES) experiments in cuprate superconductors. The energy of this kink is characteristic of a specific optical phonon mode. Furthermore, a peculiar isotope-effect, manifesting in a shift of the quasiparticle dispersion at high energy upon oxygen isotope substitution, was recently measured in the high $\mathrm{T}_{c}$ materials ${ }^{5}$, undoubtedly showing that the electron-phonon interaction plays an important role in the spectral properties of cuprates.

The electron-phonon interaction has been investigated in a variety of models 6.7 .8 . Although the ground state properties of these models received great attention, less was paid to the spectral features. Noticeable exceptions include the Holstein ${ }^{9.10}$ and the Frölich ${ }^{11}$ models which are characterized by a momentum independent or a weakly momentum dependent electron-phonon coupling. However, in many systems, such as cuprates superconductors or organic materials, the electron-phonon coupling is strongly momentum dependent. This can give rise to distinctive properties of the single particle spectral features, as we will discuss in this paper. In particular we will consider a sinusoidal dependence of the coupling on the phonon momentum, which is realized in many systems including the SSH coupling in polyacetilens 7 and the coupling to the breathing mode in cuprate superconductors 12.13 .14 .

The treatment of a multi-electron system coupled with phonons is an extremely complex and difficult problem.
In general, phonons mediate an effective attraction between electrons and consequently the system becomes susceptible to various kinds of instabilities ${ }^{15,16}$, which might have a significant effect on the photoemission spectra. However, in this paper we investigate the effect of phonons on a single quasiparticle only, thus neglecting electron-electron or electron-hole scattering processes at the Fermi surface. We believe this to be suitable in describing the properties at energy or temperature scales larger than those associated with the ground state instability, e.g. the normal state of superconductors.

In fact, as different investigations have shown ${ }^{10,17}$, the polaron models, which consist of a single electron interacting with phonons ${ }^{18}$, capture much of the physics seen in the ARPES experiments on materials with significant electron-phonon interaction ${ }^{2.19}$. This includes the kink in the quasiparticle dispersion observed at the phonon characteristic frequency. One expects that the single particle description of the influence of electron-phonon coupling would be valid to describe the photoelectron or inverse photoelectron spectral function in insulators and semiconductors in which the bands are either full or empty. However things are more complicated for a strongly correlated insulator in which the conductivity gap is a result of electron correlations and the material is an insulator in spite of having a half filled band. In systems described by the Hubbard or t-J models the single hole spectral function will be influenced by the interaction with spin fluctuations. This results in strongly dressed quasiparticles even without the electron-phonon coupling, as calculations employing self consistent Born approximation (SCBA $)^{20}$ and exact diagrammatic quantum Monte Carlo (QMC) ${ }^{21}$ shows. The later calculation also shows that the SCBA, which neglects all the magnon 
crossing diagrams, is a good approximation to the quasiparticle mass renormalization and its dispersion. When the phonons are considered, in the simplest approximation we can neglect all the crossing diagrams involving phonons and magnons and treat the interaction with phonons in a single particle fashion by taking the hole band dispersion as that given by the t-J model calculations. The approximations involving the polaron models under investigation here are done in this spirit.

In this paper we investigate the spectral properties of two polaron models: The one-dimensional Holstein $(\mathrm{H})$ mode $\mathrm{l}^{6}$ and a one-dimensional version of the breathing model $(\mathrm{B})^{40}$, relevant for cuprates superconductors ${ }^{22,23}$. The electron-phonon coupling in the H Hamiltonian is a constant, thus independent of the phonon momentum. The $\mathrm{B}$ model has a sinusoidal momentum dependent electron-phonon coupling, being small (large) for scattering with small (large) momentum phonons. In both cases we consider dispersionless optical phonons with frequency $\omega_{0}$. Although for the sake of simplicity our calculations are done in one dimension, the conclusions and the qualitative properties of the spectra are independent of dimensionality.

The difference between the properties of the two models emphasizes the importance of momentum dependent couplings. The specific momentum dependence of the B bare electron-phonon coupling results in an effective coupling which is an increasing function of the total polaron momentum, such that the small momentum polaron is in the weak coupling regime while the large momentum one is in the strong coupling regime. This might be germane to the peculiar behavior of the high energy quasiparticle dispersion ${ }^{5}$ or the temperature dependency of the photoemission linewidth ${ }^{24}$ in cuprate superconductors.

The method we use in our calculations is the selfconsistent Born approximation (SCBA) 25 . Although this method is an uncontrolled approximation which neglects electron-phonon vertex corrections in the self-energy calculation, the results at small coupling are in good agreement with numerically exact quantum Monte Carlo calculations. Because the contribution of configurations with an infinite number of phonons is considered, this approximation manages to capture the physics at large momentum, such as the gap and the flattening of the quasiparticle dispersion at the phonon energy ${ }^{26}$. It can easily provide information about excited states and thus determine the polaron spectral properties, unlike most QMC methods 11.27 .28 fit to calculate only the ground state properties.

The paper is organized as follows. In Sec. IIA we introduce the $\mathrm{H}$ and $\mathrm{B}$ Hamiltonians. The dimensionless electron-phonon coupling is defined in Sec. IIB The SCBA method is discussed in Sec. IIC The results are presented in Sec. III and their significance is discussed in Sec. IV] A short summary and the conclusions are given in Sec. $\mathbb{V}$

\section{FORMALISM}

\section{A. Model}

The Holstein Hamiltonian in real space is given by

$$
H_{H}=-t \sum_{<i j>}\left(c_{i}^{\dagger} c_{j}+\text { h.c. }\right)+\omega_{0} \sum_{i} b_{i}^{\dagger} b_{i}+g \sum_{i} n_{i} x_{i},
$$

where $c_{i}\left(b_{i}\right)$ is the electron (phonon) annihilation operator at site $i$. The first term describes the electron kinetic energy. The second term describes a set of independent oscillators with frequency $\omega_{0}$ at every site. The electronphonon coupling in the Holstein model is local and is described by the last term of Eq. 1 where the electron density $n_{i}=c_{i}^{\dagger} c_{i}$ couples with the lattice displacement $x_{i}=\frac{1}{\sqrt{2 M \omega_{0}}}\left(b_{i}^{\dagger}+b_{i}\right)$ with strength $g$.

Our breathing Hamiltonian is a one-dimensional version of the model which describes the coupling of ZhangRice (ZR) singlets 29 with the $\mathrm{Cu}-\mathrm{O}$ bond-stretching vibrations in high $\mathrm{T}_{c}$ superconductors ${ }^{22.23}$. In cuprates a ZR singlet is a bound state between a hole on the $\mathrm{Cu}$ and a hole on the four neighboring $\mathrm{O}$ atoms. It's energy is stabilized by the $\mathrm{Cu}-\mathrm{O}$ hybridization term and therefore is influenced by the $\mathrm{Cu}-\mathrm{O}$ distance. In our one dimensional model we consider a set of independent, in-between sites oscillators (the analogue of the $\mathrm{O}$ atoms) which modulate the charge carrier's (the analogue of the ZR singlet) on-site energy. Therefore we define the B Hamiltonian as

$$
\begin{aligned}
H_{B} & =-t \sum_{<i j>}\left(c_{i}^{\dagger} c_{j}+h . c .\right)+\omega_{0} \sum_{i} b_{i+\frac{1}{2}}^{\dagger} b_{i+\frac{1}{2}} \\
& +\quad g \sum_{i} n_{i}\left(x_{i-\frac{1}{2}}-x_{i+\frac{1}{2}}\right) .
\end{aligned}
$$

Both the $\mathrm{H}$ and the $\mathrm{B}$ model can be written in the momentum representation as

$$
\begin{array}{r}
H_{(H, B)}=\sum_{k} \epsilon(k) c_{k}^{\dagger} c_{k}+\sum_{q} \omega_{0} b_{q}^{\dagger} b_{q}+ \\
\frac{1}{\sqrt{N}} \sum_{k, q} \gamma_{(h, b)}(q) c_{k-q}^{\dagger} c_{k}\left(b_{q}^{\dagger}+b_{-q}\right)
\end{array}
$$

where

$$
\gamma_{H}(q)=\frac{g}{\sqrt{2 M \omega_{0}}}
$$

is the $\mathrm{H}$ electron-phonon coupling and

$$
\gamma_{B}(q)=-i \frac{2 g}{\sqrt{2 M \omega_{0}}} \sin \frac{q}{2}
$$

is the B electron-phonon coupling. Notice that in the momentum representation the $\mathrm{H}$ coupling is a constant and the B coupling is an increasing function of the phonon momentum for small momenta.

In Eq.11and Eq. 2] the free electron part of the Hamiltonian was introduced as a tight-binding hopping. However 
in order to study the influence of electron dispersion on the polaron properties in this paper we also employ calculations which consider different forms of electron dispersion.

\section{B. Electron-phonon coupling}

In this paper, we define the dimensionless electronphonon coupling as the ratio of the lattice deformation energy of a localized electron and the kinetic (delocalization) energy of the electron. This definition is encountered mostly in polaron studies focusing on the transition from large to small polarons ${ }^{28.30}$. For the Holstein model the deformation energy is given by (see Eq. A10)

$$
E_{p_{H}}=\frac{1}{2 M \omega_{0}} \frac{g^{2}}{\omega_{0}},
$$

and the dimensionless coupling is defined as

$$
\lambda_{H}=\frac{2 E_{p_{H}}}{W}=\frac{1}{2 M \omega_{0}} \frac{g^{2}}{t z \omega_{0}}
$$

where the half-bandwidth $W / 2$ is taken as a measure of the free electron kinetic energy. For a simple tight binding dispersion $W=2 z t$, where $z$ is the coordination number.

Analogously, for the breathing model the lattice deformation energy is (see Appendix A2

$$
E_{p_{B}}=\frac{1}{2 M \omega_{0}} \frac{z g^{2}}{\omega_{0}}
$$

and the dimensionless coupling is hence defined as

$$
\lambda_{B}=\frac{2 E_{p_{B}}}{W}=\frac{1}{2 M \omega_{0}} \frac{z g^{2}}{t z \omega_{0}} .
$$

Compared to the Holstein case, the lattice deformation energy for the breathing model has an extra factor of $z$ as can be seen in Eq. 8 which results form the fact that the electron interacts with $z$ neighboring oscillators.

There are several other definitions for the dimensionless electron-phonon coupling throughout the literature. For instance in the BCS theory the definition of the dimensionless coupling is $\lambda=V N(0)$, where $V$ and $N(0)$ are the effective electron-electron attraction and respectively the density of states at the Fermi level. In the weak coupling regime the effective interaction at small frequency or in the antiadiabatic limit $\left(\omega_{0} / t \rightarrow \infty\right)$ is

$$
V\left(q, \omega \ll \omega_{0}\right)=\frac{2|\gamma(q)|^{2}}{\omega_{0}} .
$$

If the integration over all $q$ momenta is considered for the breathing model, then

$$
\begin{aligned}
& \lambda_{H}^{B C S}=\quad \frac{2 N(0)}{2 M \omega_{0}} \frac{g^{2}}{\omega_{0}} \\
& \lambda_{B}^{B C S}=\frac{2 N(0)}{\omega_{0}} \frac{1}{2 \pi} \int d q|\gamma(q)|^{2}=\frac{2 N(0)}{2 M \omega_{0}} \frac{2 g^{2}}{\omega_{0}} .
\end{aligned}
$$

When choosing the density of states $N(0)=1 / W$, this definition of the dimensionless coupling coincides with our definition.

In the Migdal-Eliashberg theory of superconductivity, $\lambda^{M E}=2 \alpha^{2} F\left(\omega_{0}\right) / \omega_{0}$ where only scattering processes at the Fermi surface are considered ${ }^{31.32}$ in $\alpha^{2} F(\omega)$. This definition is equivalent to ${ }^{32}$

$$
\lambda^{M E}=\frac{m *}{m}-1
$$

where $m *$ is the quasiparticle renormalized effective mass at the Fermi surface. $\lambda^{M E}$ can be directly determined from experiments since $\alpha^{2} F(\omega)$ and $m * / m$ can be measured in tunneling ${ }^{33}$, neutron ${ }^{34}$ or respectively in the ARPES experiments ${ }^{2}$. In our case, the effective mass at the bottom of the band (which is the zero energy state of our system and in many respects similar to the Fermi surface in a many-electron system) can be determined from the equation

$(E(k)-E(0))\left(1-\frac{\partial \Sigma}{\partial \omega}(0, E(0))=\epsilon(k)-\epsilon(0)+\frac{\partial \Sigma}{\partial k}(0, E(0)) k\right.$

valid at small $k$. The ratio of the effective mass and the un-renormalized mass is

$$
\frac{m}{m *}=\frac{E(k)-E(0)}{\epsilon(k)-\epsilon(0)}=\frac{1}{1-\frac{\partial \Sigma}{\partial \omega}(0, E(0))}=Z_{0} .
$$

Therefore, we have

$$
\lambda^{M E}=\frac{1}{Z_{0}}-1 \approx-\frac{\partial \Sigma}{\partial \omega}(0, E(0)) .
$$

This definition directly relates the quasiparticle weight $Z_{0}$ to the coupling constant $\lambda^{M E}$. In the first order perturbation theory one gets

$$
\lambda^{M E}=\frac{1}{2 \pi} \int d q \frac{|\gamma(q)|^{2}}{\left(\epsilon(0)-\omega_{0}-\epsilon(q)\right)^{2}} .
$$

By inspecting Eq.17one can see that the most important contribution to the integral comes form small $q$ where the denominator is small. For the $\mathrm{B}$ model this will introduce a significant difference between $\lambda_{B}^{M E}$ and our $\lambda_{B}$ (Eq. 9). Unlike our definition which assumes average over all possible phonon momenta, $\lambda_{B}^{M E}$ is determined by small $q$ phonon scatterings which are characterized by small $\gamma(q)$ in the B model.

The definition of $\lambda^{M E}$ used in the Migdal-Eliashberg theory was intended to describe the properties at the Fermi level or at small energy excitation. It does not properly describe the physics at larger energy since the most relevant scattering processes in this case imply larger phonon momenta as we will discuss in the next two sections.

\section{Method}

In the SCBA the electron self-energy is obtained by summing over all non-crossing diagrams. In this approx- 


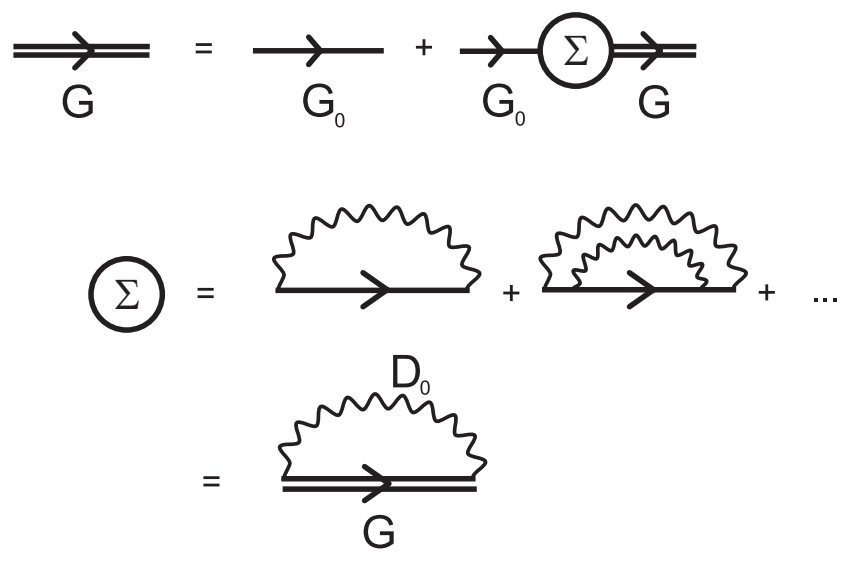

FIG. 1: Schematic representation of the Dyson equation (upper). In the SCBA approximation the self-energy is a summation of all non-crossing diagrams (middle) and can be determined self-consistently using Eq. 20 (bottom).

imation the calculation of the self-energy can be reduced to the following set of equations:

$$
\begin{gathered}
G(k, \omega)=\frac{1}{\omega-\epsilon(k)-\Sigma(k, \omega)}, \\
D(q, \Omega)=\frac{2 \omega_{0}}{\Omega^{2}-\omega_{0}^{2}},
\end{gathered}
$$

and

$$
\Sigma(k, \omega)=\frac{1}{(2 \pi)^{2}} \int \mathrm{d} \Omega \int \mathrm{d} q|\gamma(q)|^{2} D(q, \Omega) G(k-q, \omega-\Omega),
$$

where $G(k, \omega)$ and $D(q, \Omega)$ are the free electron and respectively the free phonon Green's functions (see also Fig. (1). The frequency integration in Eq. 20 can be explicitly completed and one gets

$$
\Sigma(k, \omega)=\frac{1}{2 \pi} \int \mathrm{d} q \frac{|\gamma(q)|^{2}}{\omega-\omega_{0}-\epsilon(k-q)-\Sigma\left(k-q, \omega-\omega_{0}\right)}
$$

where $\omega_{0}$ is the phonon frequency. Eq. 21] is solved selfconsistently and the spectral function is determined as $A(k, \omega)=-\frac{1}{\pi} \operatorname{Im} G(k, \omega)$. The overlap of the polaron state $\mid \nu_{k}>$ (the lowest energy state at a particular momentum $k$ which in general is an isolated pole in the Green's function) with the free electron state, $c_{k}^{\dagger}|0\rangle$

$$
Z_{k}=\left|\left\langle\nu_{k}\left|c_{k}^{\dagger}\right| 0\right\rangle\right|^{2},
$$

is called the quasiparticle weight and can be calculated $\operatorname{as}^{25}$

$$
Z_{k}=\left.\frac{1}{1-\frac{\partial \Sigma(k, \omega)}{\partial \omega}}\right|_{\omega=E_{k}}
$$

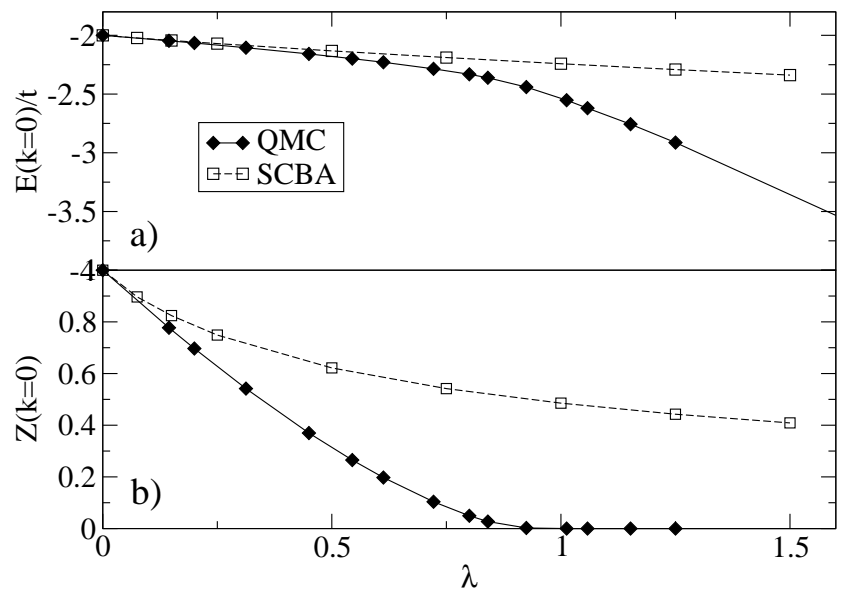

FIG. 2: Numerically exact QMC (diamonds) and SCBA (squares) results for polaron energy $E_{0}$ (a) and quasiparticle weight $Z_{0}$ (b) versus the dimensionless electron-phonon coupling $\lambda$ (see Eq. 7) at $k=0$. Holstein model with $t=1$ and $\omega_{0}=0.1 t$ and $2 M=1$.

The neglect of vertex corrections in the SCBA results in a failure of this approximation in the intermediate and strong coupling regime. This is apparent in Fig. 2 where a comparison of SCBA and exact QMC results for the $\mathrm{H}$ model energy and quasiparticle weight at $k=0$ is shown. In fact the polaron properties at $k=0$ are better approximated in a regular Rayleigh-Schrodinger (RS) perturbation theory than in $\mathrm{SCBA}^{35}$. However, at large $k$ where the polaron energy approaches the phonon frequency, the RS perturbation theory fails and the SCBA which considers configurations involving a large number of phonons provides good results. In order to show that, we compare the SCBA results with the exact ones obtained by using the diagrammatic quantum Monte Carlo technique ${ }^{36.37}$ in Fig. 3 As can be seen in Fig. 3-a, the dispersion becomes flat when the polaron energy gets close to the the phonon frequency $E_{0}+\omega_{0}, E_{0}$ being the bottom of the polaronic band. The flattening is accompanied by a strong reduction of the quasiparticle weight, as shown in Fig. 3 -b. The SCBA slightly underestimates the energy corrections in the flat dispersion region but otherwise captures all these features successfully.

The $\mathrm{H}$ polaron physics at small coupling has been investigated with different techniques for many years and is rather well understood now. The polaron character changes from almost free electron at the bottom of the band to a one phonon plus one electron state in the flat dispersion region where almost all the momentum is carried by the phonon. The states below the one phonon threshold $E_{0}+\omega_{0}$ are bound states ${ }^{11}$, characterized by delta peaks in the spectral representation. Above it, there is a continuum of states and the electron self-energy acquires a finite imaginary part. However, at large energies relative to $\omega_{0}$ the self-energy's imaginary part is small and the spectral representation is characterized by sharp peaks with a dispersion close to the free electron 

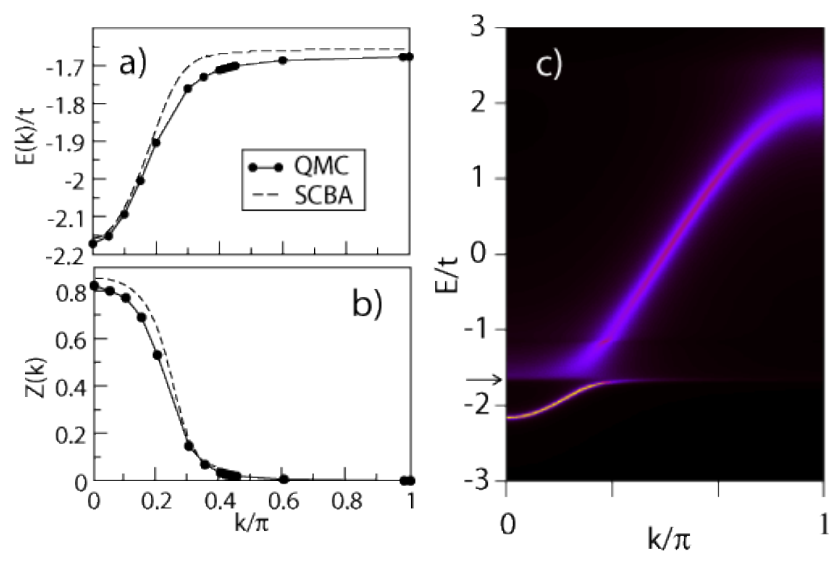

FIG. 3: Holstein polaron dispersion $E(k)$ (a) and quasiparticle weight $Z(k)$ (b) calculated with SCBA (dotted line) and QMC (circles). c) Spectral representation obtained with SCBA. Everywhere $\lambda_{H}=0.25$ and $\omega_{0}=0.5 t$.

one. In a photoemission experiment the dispersion of the high intensity peak would exhibit something resembling a kink, although a small gap at the phonon energy and sudden onset of broadening appears at the same time. The spectral function at momenta corresponding to energies well below the phonon frequency will consist of a sharp peak followed by a broad satellite, such as it is observed in surface studies of $B e^{19}$ for instance. This can be seen in Fig. 3 c) where a false color plot of spectral intensity obtained with SCBA is shown (see also Fig. 8 -a). The physics discussed in this paragraph (i.e. the dispersion flattening and the gap in the polaron spectrum) is presumably true even for very small electron-phonon couplings. However, for such small couplings these features are too small to be captured experimentally or even numerically.

\section{RESULTS}

In our calculations we take $t=1$ and $2 M=1$. For the one-dimensional case the coordination number $z=2$.

In Fig. 4 -a and -b we show the polaron energy $E_{0}$ and respectively the quasiparticle weight $Z_{0}$ versus the dimensionless coupling constant $\lambda$ at $k=0$. For the $\mathrm{H}$ model, the decrease of $Z_{0}$ with increasing $\lambda$ is not very different from the one given by the ME theory (dashed line in Fig. (4. given by Eq. 16), showing that the ME definition of $\lambda$ is similar to ours. However, for the B model the quasiparticle weight $Z_{0}$ and the polaron energy $E_{0}$ decrease much slower with increasing $\lambda$. As mentioned in the previous section this is due to the small momentum scattering processes implied in the renormalization of $Z_{0}$ (see Eq. 17) and, similarly, in the determination of the self-energy. It is worth pointing out that, unlike the $\mathrm{H}$ polaron, even for values of $\lambda \approx 1$, the $\mathrm{B}$ polaron remains in the weak coupling regime and hence the difference between the fully convergent SCBA (full symbols) and the

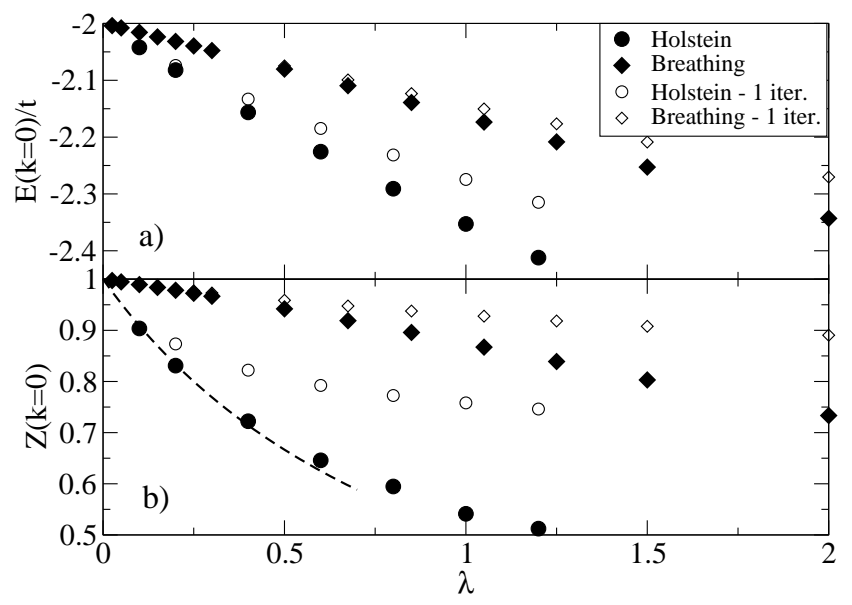

FIG. 4: H (circles) and B (diamonds) polaron energy (a) and quasiparticle weight (b) versus $\lambda$ at $k=0$ and $\omega_{0}=0.2 t$. The open symbols are the results obtained after only one iteration (first order in $D$ ). The dashed line in (b) corresponds to $\lambda^{M E}=\frac{1}{Z_{0}}-1$ (see Eq. [16).
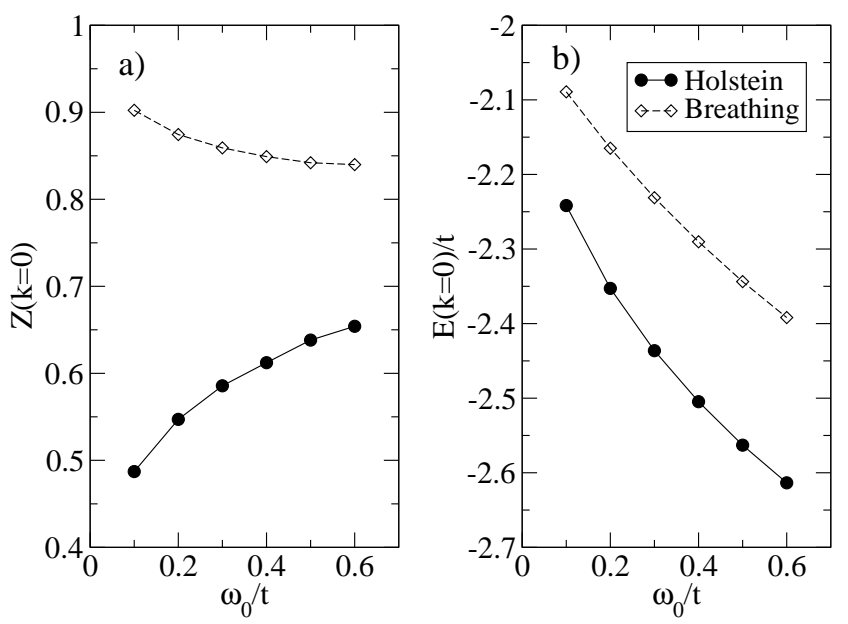

FIG. 5: The quasiparticle weight $Z_{0}$ (a) and the polaron energy $E_{0}(\mathrm{~b})$ at zero momentum versus phonon frequency $\omega_{0}$ for the $\mathrm{H}$ and $\mathrm{B}$ models at $\lambda_{H}=0.2$ and respectively $\lambda_{B}=1.3$.

first order perturbation theory (i.e. only the first SCBA iteration, empty symbols) is small. Another interesting feature is that for the same value of $Z_{0}$ the $\mathrm{B}$ polaron energy is lower than the $\mathrm{H}$ one, showing that the ratio between the energy renormalization and the quasiparticle weight renormalization is different for the two models.

Another important difference between the two models is the dependence of the polaron properties at the band bottom on the phonon frequency $\omega_{0}$. While for the $\mathrm{H}$ case an increase of $\omega_{0}$ results in an increase of the quasiparticle weight $Z_{0}$ the opposite behavior is seen for the $\mathrm{B}$ model. This is illustrated in Fig. 5. 5 . The reason for the reduction of $Z_{0}$ with increasing $\omega_{0}$ in the $\mathrm{B}$ model can be easily understood by noticing (see Eq. 17) that a larger value of $\omega_{0}$ reduces the importance of the $q$ dependence in 

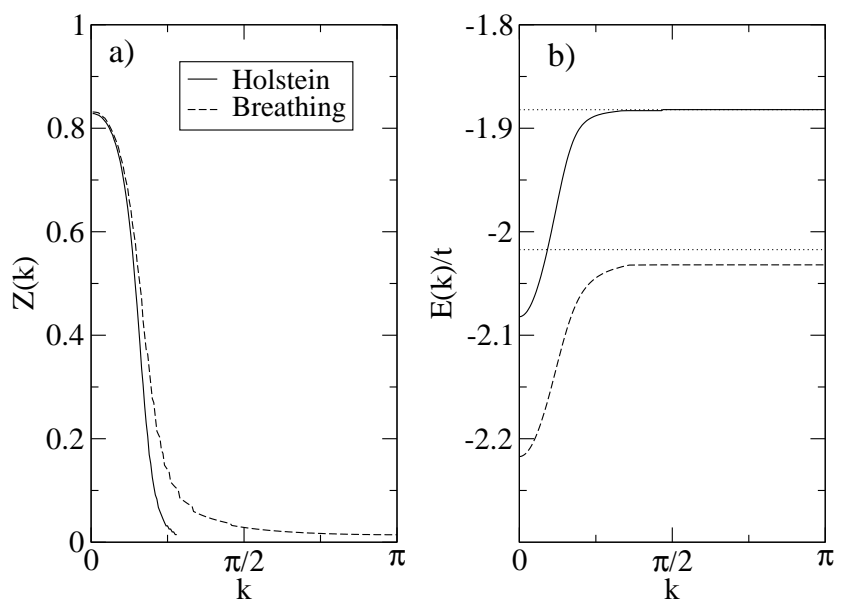

FIG. 6: Quasiparticle weight $Z(k)$ (a) and the polaron energy $E(k)$ (b) for the $\mathrm{H}$ and $\mathrm{B}$ model for $\omega_{0}=0.2 t$ at coupling $\lambda_{H}=0.2$ and $\lambda_{B}=1.3$ respectively. The dotted horizontal lines in (b) marks the first phonon threshold energy $E_{0}+\omega_{0}$.

the polaron properties calculation. As discussed earlier, the strong momentum dependent coupling is responsible for the weak $Z_{0}$ renormalization of the $\mathrm{B}$ polaron and thus an increase of $\omega_{0}$ would result in a larger effective coupling and implicitly in a smaller $Z_{0}$.

Aside from the different $\lambda$ and $\omega_{0}$ dependency of the two models at zero momentum, the momentum dependent properties also exhibit different behaviors. This is shown in Figs. 6 7,8 and 9 where the $k$ dependent properties for the two models are illustrated.

In Fig. [6 and Fig. [7 a and -b we have chosen the value of $\lambda$ such that both models yield the same quasiparticle weight at the bottom of the band. Thus, the choice of $\lambda_{H}=0.2$ and $\lambda_{B}=1.3$ results in $Z_{0 h}=Z_{0 b}=0.83$, implying that both models are in the weak coupling regime. As mentioned earlier, the B polaron energy at $k=0$ is lower. At large $k$, just below the first phonon threshold energy $E_{0}+\omega_{0}$, both polarons display a flat dispersion and a reduced quasiparticle weight. However, the $\mathrm{B}$ polaron quasiparticle weight at large $k$ is substantially larger (for instance $Z_{B}(k=\pi) \approx 0.014$ within a numerical precision of $10^{-3}$ and $\left.Z_{H}(k=\pi)<10^{-3}\right)$, making the $\mathrm{B}$ polaron state at large $k$ distinguishable in the spectral plot (Fig. 7-b) in contrast to the $\mathrm{H}$ one (Fig. [7-a). At energies larger than $E_{0}+\omega_{0}$ the spectral intensity of the $\mathrm{B}$ quasiparticle is much smaller than the $\mathrm{H}$ model one, unlike the situation at the band bottom where both models have the same $Z_{0}$. This large momentum behavior points to a stronger effective coupling for the B model at large $k$.

While the dispersion of both models displays a gap at $E_{0}+\omega_{0}$, the B polaron shows a second gap at the second phonons threshold energy $E_{0}+2 \omega_{0}$. This can be seen in Fig. 17-b but also occurs for smaller values of the dimensionless coupling as shown in Fig. [7-c for the value of $\lambda_{B}=0.5$. This value was chosen such that the ground state energy $E_{0}$ of the $\mathrm{B}$ model is equal to that of the Hol-
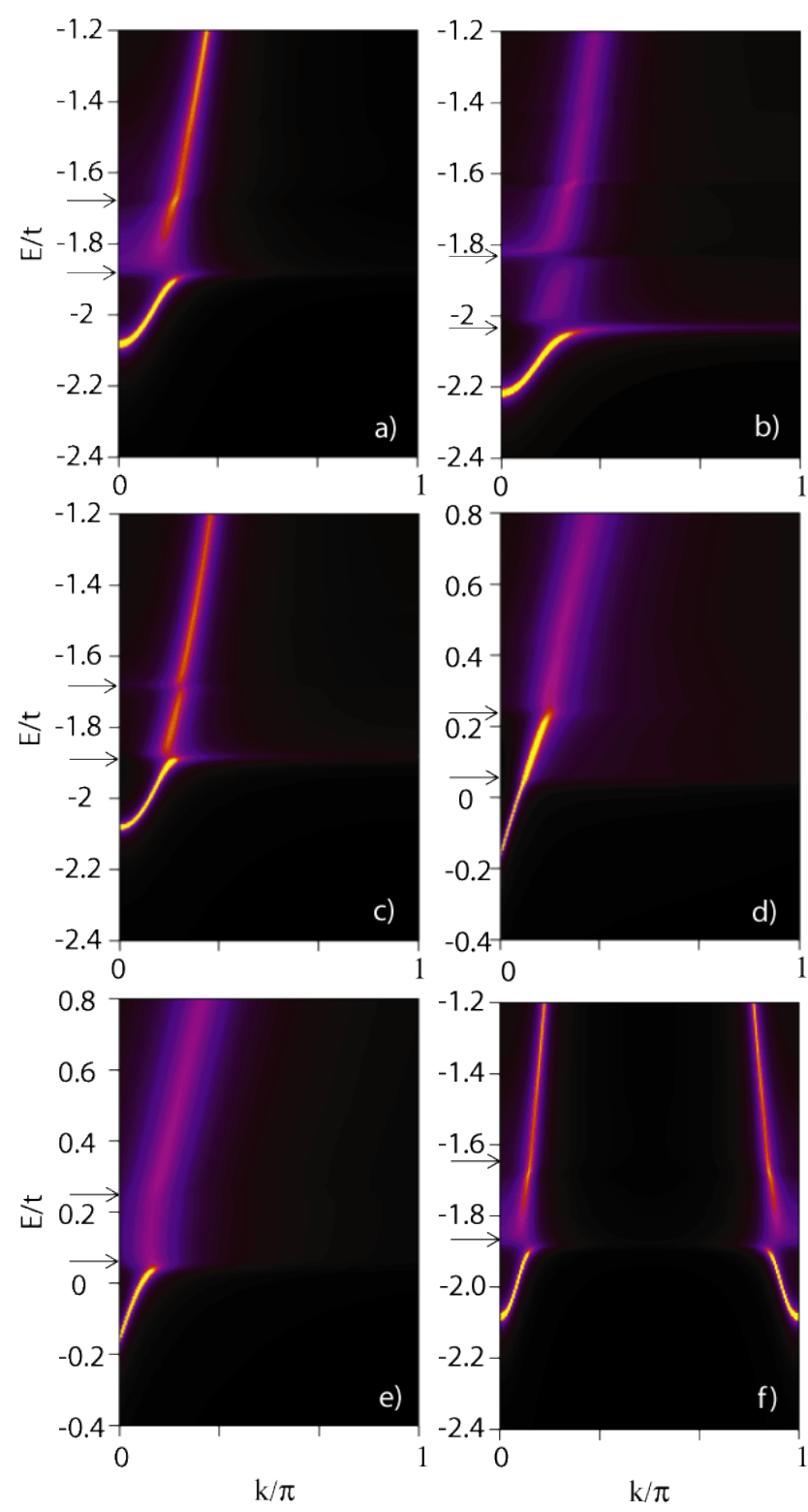

FIG. 7: Spectral representation for: a) $\mathrm{H}$ model, $\lambda_{H}=0.2$, tight binding dispersion, b) B model, $\lambda_{B}=1.3$, tight binding dispersion, c) B model, $\lambda_{B}=0.5$, tight binding dispersion, d) $\mathrm{B}$ model, $\lambda_{B}^{\text {lin }}=1.6$, linear dispersion with $v_{F}=t$ (Eq. 25), e) $\mathrm{H}$ model, $\lambda_{H}^{\text {lin }}=0.9$, linear dispersion with $\left.v_{F}=t, \mathrm{f}\right)$ $\mathrm{H}$ and $\mathrm{B}$ model, $\lambda_{H}=\lambda_{B}=0.2$, with dispersion $\epsilon(k)=$ $-2 t \cos (2 k)$. Everywhere $\omega_{0}=0.2 t$. The arrows indicate the first (lower) and the second (upper) phonon threshold energies corresponding to $E_{0}+\omega_{0}$ and respectively $E_{0}+2 \omega_{0}$.

stein polaron one shown in Fig. (7)a. The situation can be even clearer visualized by comparing Fig. 88-a with Fig. 8 $\mathrm{b}$, where the energy distribution curves (EDC) for $\mathrm{H}$ and respectively $\mathrm{B}$ cases are shown.

An even more interesting effect is noticed if a linear dispersion for the free electron is considered

$$
\epsilon(k)=v_{F}|k|,
$$




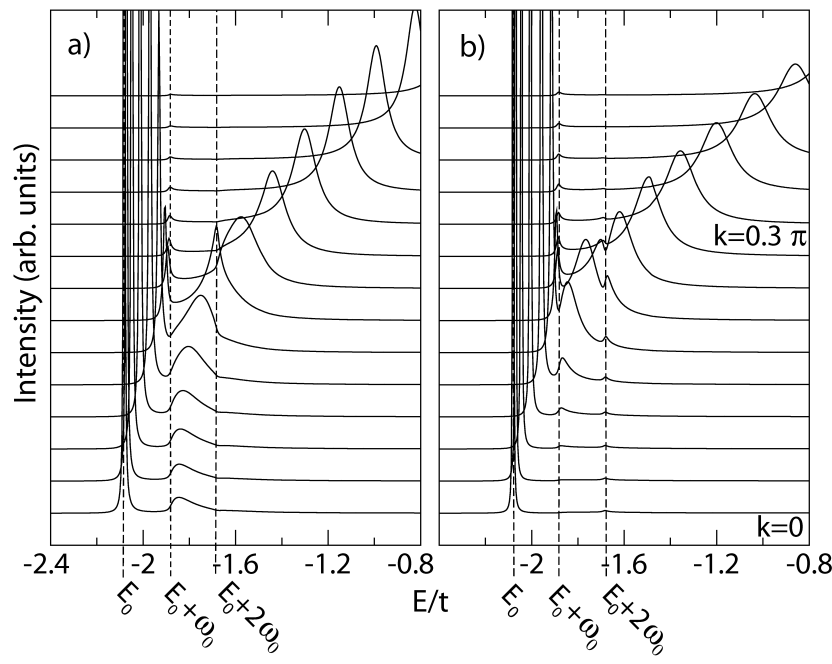

FIG. 8: Spectral function (energy distribution curves) for a) $\mathrm{H}$ model with $\lambda_{H}=0.2$ and b) $\mathrm{B}$ model with $\lambda_{B}=0.5$. Free electron tight binding dispersion and $\omega_{0}=0.2 t$ is considered.
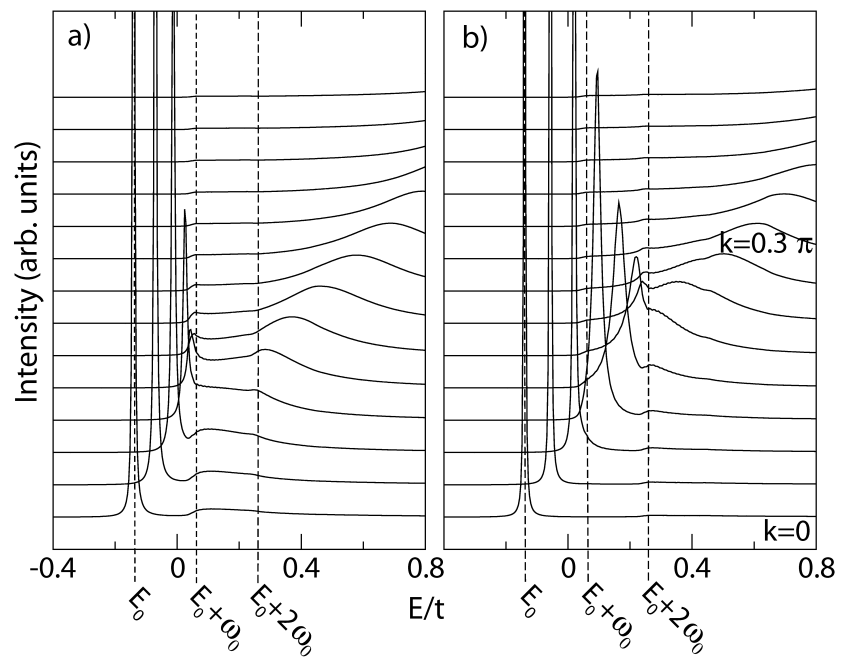

FIG. 9: Spectral function (energy distribution curves) for a) $\mathrm{H}$ model with $\lambda_{H}^{l i n}=0.9$ and $\mathrm{b}$ ) $\mathrm{B}$ model with $\lambda_{B}^{l i n}=1.6$. Free electron linear dispersion $\left(v_{F}=t\right)$ and $\omega_{0}=0.2 t$ is considered.

with a value of $v_{F}$ close to one or larger. In this case one can take $v_{F}$ to be a measure of the free electron kinetic energy ${ }^{\underline{41}}$ and thus define the dimensionless coupling as

$$
\lambda_{B}^{l i n}=\frac{E_{p_{B}}}{v_{F}}=\frac{1}{2 M \omega_{0}} \frac{2 g^{2}}{v_{F} \omega_{0}} .
$$

The resulting $\mathrm{B}$ polaron dispersion is shown in Fig. [7 $\mathrm{d}$ (see also the corresponding EDC plot in Fig. 9-b). While it displays a gap at $E_{0}+2 \omega_{0}$, no distinguishable gap or kink can be seen at the first phonon threshold energy $E_{0}+\omega_{0}$. This free-electron like behavior of the polaron at $E_{0}+\omega_{0}$ is due to the fact that the physics there is determined by very small $q$ scatterings, characterized by small coupling strength $\gamma(q)$, originating from the rapid increase of the electron energy with $k$. At larger energy, close to $E_{0}+2 \omega_{0}$, the relevant phonon momenta $q$ implied in the scattering are larger and the physics is consequently determined by a larger effective coupling. As a result a noticeable kink appears at this energy in the spectrum. This effect (i.e. kink at $E_{0}+2 \omega_{0}$ but no noticeable one at $\left.E_{0}+\omega_{0}\right)$ is a result of an electron-phonon coupling which is an increasing function of the polaron momentum and it is hence not seen in the Holstein model even for the case of a linear electronic dispersion (see Fig. [7. and the corresponding EDC plot in Fig. 97a).

The differences between the two models discussed above are a consequence of two effects: i) the strong $q$ dependence of the bare electron-phonon coupling $\gamma(q)$ in the $\mathrm{B}$ model and ii) the polaron properties at small $k$ are most strongly influenced by the small momentum $q$ phonons. However the second statement is not true if the free electron dispersion has low energy states separated by large $q$ as we will discuss in the next section (Sec IV). In order to show this we choose a free electron dispersion

$$
\epsilon(k)=-2 t \cos (2 k),
$$

which is double degenerate with the lowest energy values at $k=0$ and $k=\pi$ respectively. We find that for this electronic dispersion the differences between the $\mathrm{H}$ and the B model are very small, less than $0.1 \%$, and therfore not discernible in the spectral representation plot shown in Fig. [7.f.

The general features of the polaron spectral function illustrated in Figs. 8 and 9 show a remarkable resemblance with the photoemission data in materials with significant electron-phonon interactions ${ }^{2,19}$. For momenta which correspond to energies below the phonon frequency one can see a sharp peak followed by a broad satellite. At large momenta, the broad satellite found at energies well above the phonon frequency sharpens and follows the free electron dispersion while the intensity of the sharp peak below $\omega_{0}$ vanishes. We want to point that this picture, with the spectral function determined by two main branches, is quite different form the one incorrectly presented in some popular textbooks ${ }^{38}$ where the spectrum is characterized by a single quasiparticle peak which changes its dispersion slope when crossing the phonon energy threshold.

\section{DISCUSSION}

For a tight-binding or a linear dispersion, the B polaron properties at small momentum are determined by small $q$ scatterings characterized by small $\gamma(q)$. Let's consider the first order self-energy diagram contribution

$$
\Sigma(k, \omega)=\frac{1}{2 \pi} \int d q \frac{|\gamma(q)|^{2}}{\omega-\omega_{0}-\epsilon(k-q)} .
$$


The most significant contribution below the one phonon threshold, i.e. when $\omega<\epsilon(0)+\omega_{0}$, is given by the values of transferred momentum $q$ for which $\epsilon(k-q)$ is minimum which implies $q \approx k$. The value of $k$ where the polaron energy reaches the one-phonon threshold is small $\left(k_{1}\right.$ in Fig. 10-a) and thus, the relevant scatterings which determine the physics below $E_{0}+\omega_{0}$ occur at small momentum $q$, implying a small effective coupling.

For a linear dispersion with $v_{F} \approx 1$ or larger, the value of $k$ where the first phonon threshold energy is reached is even smaller. Besides, the electronic density of states at small momentum, which is proportional to the number of relevant scatterings, is also much smaller then for a 1D tight-binding dispersion ${ }^{42}$. These two conditions yield a very small effective coupling at the first phonon threshold. Consequently, even for large values of $\lambda$ as defined in Eq. 25] the polaron dispersion exhibits an extremely narrow gap, hardly discernible in our calculation.

The B model properties at larger momentum indicate an increase of the effective electron-phonon coupling with increasing the polaron momentum, due to the increase of the momentum of the relevant scattered phonons. For example, the most significant scattering at the two phonon threshold $\left(\omega \approx E_{0}+2 \omega_{0}\right)$ are those for which $\epsilon(k-q) \approx \omega_{0}$. If $k_{1}$ is the value of the electron momentum for which $\epsilon\left(k_{1}\right)=\epsilon\left(-k_{1}\right)=\omega_{0}$ the resulting values for the phonon momentum $q$ relevant in the scattering are $q \approx k \pm k_{1}$. The larger $q$ solution, i.e. $q \approx k+k_{1}\left(k_{2}\right.$ in Fig. 10-a), implies a larger effective coupling.

A similar analysis of the higher order self-energy diagrams leads to the same conclusion: The effective coupling in the B polaron model is increasing with momentum, and hence the small energy and momentum B polaron is characterized by a small effective coupling while the high energy and large momentum properties are determined by a large effective coupling. Moreover, at low energy the contribution of the crossing diagrams is comparable to the contribution of the crossing diagrams in a $\mathrm{H}$ model at small coupling. Thus, the SCBA solution is a good approximation for the low energy, small $k$, polaron. Nevertheless, the large momentum properties are characterized by large effective couplings and hence the SCBA approximation is questionable in that region.

The conclusions based on the analysis of self-energy diagrams discussed above implicitly assume that a small $\epsilon(k-q)$ implies small $q$, when the polaron momentum $k$ is small. However, as we have shown in the previous section, if the electron dispersion has low energy states separated by large $q$ the above assumption is invalid. Thus the effective coupling at small energy is substantially larger and besides there is no significant increase of the effective coupling with polaron momentum or energy.

For example the strong dependence of the effective coupling on the momentum does not hold in a metallic system with a large Fermi surface. To see this, one can think of the Fermi surface as a degenerate ground state for the quasiparticle $\frac{43}{3}$. Unlike the non-degenerate ground state case where the small energy scattering is restricted to

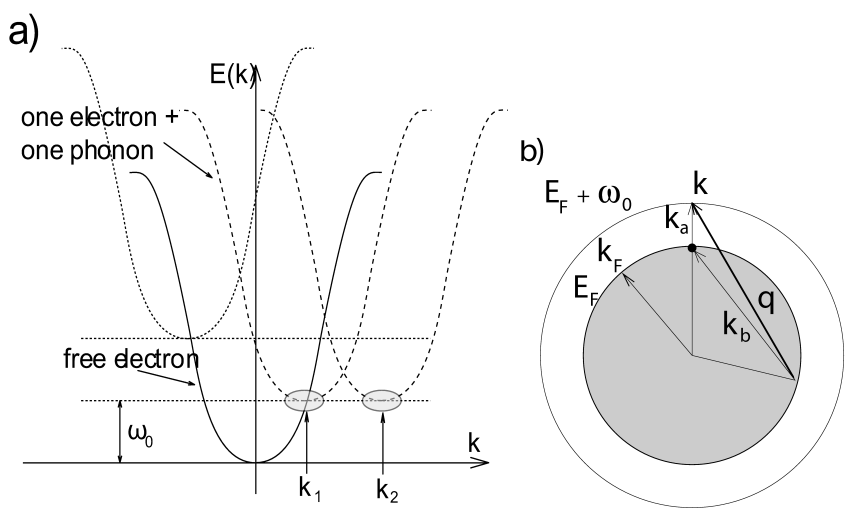

FIG. 10: a) Energy levels for zero coupling $(g=0)$. The solid line shows the non-interacting electron dispersion, the dashed ones are one phonon + one electron states and the dotted one two phonons + one electron, etc. $k_{1}$ is the momentum where the electron dispersion reaches $\omega_{0}$ and has the most significant contribution to the scattering at the first phonon threshold. $k_{2}$ is the value of momentum with significant contribution to the scattering at the second phonon threshold. b) For the polaron with momentum $k$ the scattering with momentum $q$ has a significant contribution. Although the polaron momentum is close to $k_{F}$, thus $k_{a}=k-k_{F}$ is small, $q=k_{a}+k_{b}$ is large because $k_{b}$ which connects two points on the Fermi surface can be large.

small momentum, the relevant scatterings in the presence of a Fermi surface are restricted to small momentum plus a vector which connects two points on the Fermi surface $\left(q=k_{a}+k_{b}\right.$ in Fig. 10 b)). Therefore scatterings with large momentum, for which $\gamma(q)$ is large, are relevant. This makes the effective coupling to be large even at small energy. Because now at both low energy and high energy there are relevant large momentum scatterings the dependence of the effective coupling strength on the energy and momentum will be modest.

Although dimensionality plays an important role in determining the quantitative polaron properties ${ }^{28.30}$, the main features of the $\mathrm{B}$ polaron are a consequence of strong momentum dependence of the bare electronphonon coupling. Therefore the main conclusions of this study remain valid for $2 \mathrm{D}$ or $3 \mathrm{D}$ systems as long as the electron-phonon coupling has a similar $q$ dependence (increases with $q$ ).

The particularities of the B polaron discussed in this paper might be relevant for the weakly doped cuprates characterized by very small Fermi pockets around the $( \pm \pi / 2, \pm \pi / 2)$ points in the Brillouin Zone which makes the situation very similar to the one captured by the polaron model. The quasiparticles in this system have a four-fold degenerate ground state with the states separated by $(0, \pi)$ or $(\pi, 0)$ vectors ${ }^{20}$. The relevant phonon mode is believed to be the half-breathing one for which the electron-phonon coupling has a strong $q$ dependence, being small at small $q$ and large at $(0, \pi)$. If for some reasons the scattering between the $( \pm \pi / 2, \pm \pi / 2)$ points is restricted or if its importance is small because of an addi- 
tional $k$ dependence in the bare electron-phonon coupling $\gamma(k, q)$ the formed polarons will be characterized by a momentum and energy dependent effective coupling. Consequently, this implies interesting energy and temperature dependent properties such as a strongly temperature dependent quasiparticle photoemission linewidth 24 . However, if the scattering between the $( \pm \pi / 2, \pm \pi / 2)$ states is relevant, the $q$ dependence of the coupling will not be very important and presumably a Holstein like coupling can be used to describe the physics as well, analogous to the 1D case discussed in Fig. [7.f.

\section{CONCLUSIONS}

In this paper we study the spectral properties of the one-dimensional Holstein and breathing polaron models. While the $\mathrm{H}$ model has a momentum independent electron-phonon coupling, the B coupling is a monotonic increasing function of the phonon momentum. We find the renormalization of the quasiparticle properties at small momentum in the $\mathrm{B}$ model weaker than that in the $\mathrm{H}$ one for a dimensionless coupling constant defined as the ratio of the lattice deformation energy and the kinetic energy of the electron. With increasing phonon frequency $\omega_{0}$, the B quasiparticle weight decreases in contrast to the $\mathrm{H}$ case where it increases. The quasiparticle dispersion in the $\mathrm{H}$ model displays a kink and a small gap at an excitation energy equal to $\omega_{0}$. On the other hand, the $\mathrm{B}$ model dispersion, besides the gap at $\omega_{0}$, exhibits another, more pronounced one at energy $2 \omega_{0}$. This is due to the following facts: the momentum $q$ of the relevant phonons in the scattering process increases with increasing the total polaron momentum and that the $\mathrm{B}$ coupling is an increasing function of $q$. As a consequence the renormalization of the $\mathrm{B}$ polaron increases with increasing polaron momentum. However the first fact is dependent on the free electron dispersion and does not hold if it has low energy states separated by large momentum. In that case the difference between the $\mathrm{B}$ and the $\mathrm{H}$ polaron becomes less significant.

The specific momentum dependence of the B bare electron-phonon coupling results in an effective coupling which is an increasing function of the total polaron momentum, such that the small momentum polaron properties are weak coupling while the large momentum ones are strong coupling. This might be relevant for explaining the peculiar behavior of the high energy quasiparticle dispersion ${ }^{5}$ or the temperature dependency of the photoemission linewidth ${ }^{24}$ in cuprate superconductors.

\section{Acknowledgments}

The work was supported by NSF grants DMR-0312680 and DMR-0113574, and by CMSN grant DOE DEFG02-04ER46129. We acknowledge Ohio Supercomputer Center where part of the computation was performed.
G. A. Sawatzky acknowledges the financial support from the Canadian funding organizations NSERC, CIAR and CFI. Thomas Maier acknowledges support from the Center for Nanophase Materials Sciences, Oak Ridge National Laboratory, which is funded by the Division of Scientific User Facilities, U.S. Department of Energy.

\section{APPENDIX A: EFFECTIVE COUPLING}

\section{Holstein}

The last two terms of the Holstein Hamiltonian (Eq.11) can be diagonalized via the Lang-Firsov canonical transformation ${ }^{39}$ defined by a unitary operator $e^{S}$ where

$$
S=-\frac{g}{\omega_{0} \sqrt{2 M \omega_{0}}} \sum_{i} n_{i}\left(b_{i}^{\dagger}-b_{i}\right) .
$$

Using the expansion

$$
\tilde{A}=e^{S} A e^{-S}=A+[S, A]+\frac{1}{2}[S,[S, A]]+\ldots
$$

we find for the transformed phonon annihilation operator:

$$
\tilde{b}_{i}=b_{i}+\frac{g}{\omega_{0} \sqrt{2 M \omega_{0}}} n_{i}
$$

and similarly for the electron operator

$$
\tilde{c}_{i}=c_{i} e^{\frac{q}{\omega_{0} \sqrt{2 M \omega_{0}}}\left(b_{i}^{\dagger}-b_{i}\right)} .
$$

The tilde mark is used to label the transformed operators. The Holstein Hamiltonian in the new basis can be written as

$$
\begin{gathered}
H=H_{0}+H_{t} \\
H_{0}=\sum_{i} \omega_{0} \tilde{b}_{i}^{\dagger} \tilde{b}_{i}-\frac{g^{2}}{2 M \omega_{0}^{2}} \sum_{i} \tilde{n}_{i}^{2} \\
H_{t}=-t \sum_{<i j>}\left(\tilde{c}_{i}^{\dagger} \tilde{c}_{j} X_{i}^{\dagger} X_{j}+H . c .\right)
\end{gathered}
$$

where

$$
X_{i}=e^{-\frac{g}{\omega_{0} \sqrt{2 M \omega_{0}}}\left(\tilde{b}_{i}^{\dagger}-\tilde{b}_{i}\right)} .
$$

For a single electron the density term in Eq. A6 simplifies, yielding

$$
H_{0}=\sum_{i} \omega_{0} \tilde{b}_{i}^{\dagger} \tilde{b}_{i}-\frac{g^{2}}{2 M \omega_{0}^{2}} \sum_{i} \tilde{n}_{i}
$$


One can define a dimensionless coupling constant for the electron-phonon interaction as the ratio between the gained lattice deformation energy (see second term in $H_{0}$ )

$$
E_{p_{H}}=\frac{1}{2 M \omega_{0}} \frac{g^{2}}{\omega_{0}}
$$

and the bare electron kinetic energy, taken to be the halfbandwidth, $W / 2=z t$,

$$
\lambda_{H}=\frac{2 E_{p_{H}}}{W}=\frac{1}{2 M \omega_{0}} \frac{g^{2}}{z \omega_{0} t} .
$$

\section{Breathing}

The electron-phonon interaction term in the B Hamiltonian (see last term in Eq. 22) can, by changing the order of summation, be re-write it in the form

$$
H_{\text {int }}=g \sum_{i} x_{i-\frac{1}{2}}\left(n_{i}-n_{i-1}\right),
$$

where $x_{i}$ is the lattice displacement. The last two parts of the Hamiltonian are once again diagonalized via the unitary operator $e^{S}$ where

$$
S=-\frac{g}{\omega_{0} \sqrt{2 M \omega_{0}}} \sum_{i} n_{i}\left(b_{i-\frac{1}{2}}^{\dagger}-b_{i-\frac{1}{2}}\right) .
$$

Using the expansion of Eq. A2 we find for the transformed phonon and electron annihilation operators:

$$
\begin{gathered}
\tilde{b}_{i-\frac{1}{2}}=b_{i-\frac{1}{2}}+\frac{g}{\omega_{0} \sqrt{2 M \omega_{0}}}\left(n_{i}-n_{i-1}\right) \\
\tilde{c}_{i}=c_{i} e^{\frac{g}{\omega_{0} \sqrt{2 M \omega_{0}}}\left(b_{i-\frac{1}{2}}^{\dagger}-b_{i-\frac{1}{2}}\right)} .
\end{gathered}
$$

Substituting these transformed operators we find in the new basis

$$
\begin{aligned}
\tilde{x}_{i-\frac{1}{2}} & =\frac{1}{\sqrt{2 M \omega_{0}}}\left(\tilde{b}_{i-\frac{1}{2}}^{\dagger}+\tilde{b}_{i-\frac{1}{2}}\right) \\
& =\frac{1}{\sqrt{2 M \omega_{0}}}\left(b_{i-\frac{1}{2}}^{\dagger}+b_{i-\frac{1}{2}}\right)+\frac{2 g}{2 M \omega_{0}^{2}}\left(n_{i}-n_{i-1}\right)
\end{aligned}
$$

1 T. Cuk, D. H. Lu, X. J. Zhou, Z.-X. Shen, T. P. Devereaux, and N. Nagaosa, Phys. Stat. Sol. 242, 11 (2005).

2 A. Lanzara, P. V. Bogdanov, X. J. Zhou, S. A. Kellar, D. L. Feng, E. D. Lu, T. Yoshida, H. Eisaki, A. Fujimori, K. Kishio, et al., Nature 412, 510 (2001).

3 P. V. Bogdanov, A. Lanzara, S. A. Kellar, X. J. Zhou, and for the breathing-Hamiltonian

$$
\begin{gathered}
H_{0}=\sum_{i} \omega_{0} \tilde{b}_{i}^{\dagger} \tilde{b}_{i}-\frac{g^{2}}{2 M \omega_{0}^{2}} \sum_{i}\left(\tilde{n}_{i}-\tilde{n}_{i-1}\right)^{2} \\
H_{t}=-t \sum_{<i j>}\left(\tilde{c}_{i}^{\dagger} \tilde{c}_{j} X_{i}^{\dagger} X_{j}+H . c .\right)
\end{gathered}
$$

where

$$
X_{i}=e^{-\frac{g}{\omega_{0} \sqrt{2 M \omega_{0}}}\left(\tilde{b}_{i-\frac{1}{2}}^{\dagger}-\tilde{b}_{i-\frac{1}{2}}\right)} .
$$

In the case of a system only containing a single electron the density terms contained in Eq. A17 simplify, yielding

$$
H_{0}=\sum_{i} \omega_{0} \tilde{b}_{i}^{\dagger} \tilde{b}_{i}-\frac{2 g^{2}}{2 M \omega_{0}^{2}} \sum_{i} \tilde{n}_{i}
$$

Hence, the lattice deformation energy is found to be

$$
E_{p_{B}}=\frac{1}{2 M \omega_{0}} \frac{2 g^{2}}{\omega_{0}}
$$

In contrast to the $\mathrm{H}$ model (Eq. A10) we incur an additional factor of 2 due to a coupling with two neighboring oscillators. For higher dimensionality the number of neighboring oscillators is given by the coordination number $z$, thus replacing the factor 2 in Eq. A21 with $z$.

We thus define the dimensionless coupling constant for the electron-breathing-phonon interaction

$$
\lambda_{B}=\frac{2 E_{p_{B}}}{W}=\frac{1}{2 M \omega_{0}} \frac{z g^{2}}{z \omega_{0} t} .
$$

This definition is identical to that found for the Holstein polaron besides the overall factor of $z$.
E. D. Lu, W. J. Zheng, G. Gu, J.-I. Shimoyama, K. Kishio, H. Ikeda, et al., Phys. Rev. Lett. 85, 2581 (2000).

4 A. Kaminski, M. Randeria, J. C. Campuzano, M. R. Norman, H. Fretwell, J. Mesot, T. Sato, T. Takahashi, and K. Kadowaki, Phys. Rev. Lett. 86, 1070 (2001).

${ }^{5}$ G.-H. Gweon, T. Sasagawa, S. Y. Zhou, J. Graf, H. Takagi, 
D.-H. Lee, and A. Lanzara, Nature 430, 187 (2004).

6 T. Holstein, Annals of Phys. 8, 325 (1959).

7 W. P. Su, J. R. Schrieffer, and A. J. Heeger, Phys. Rev. Lett. 42, 1698 (1979).

${ }^{8}$ h. H. Fröhlich, H. Pelzer, and S. Zienau, Philos. Mag. 41, 221 (1950).

9 S. Engelsberg and J. R. Schrieffer, Phys. Rev. 131, 993 (1963).

10 A. S. Mishchenko and N. Nagaosa, Phys. Rev. Lett. 93, 036402 (2004).

11 N. V. Prokof'ev and B. V. Svistunov, Phys. Rev. Lett. 81, 2514 (1998).

12 A. Abanov, A. V. Chubukov, M. Eschrig, M. R. Norman, and J. Schmalian, Phys. Rev. Lett. 89, 177002 (2002).

13 B. Friedl, C. Thomsen, and M. Cardona, Phys. Rev. Lett. 65, 915 (1990).

14 H.-Y. Kee, S. A. Kivelson, and G. Aeppli, Phys. Rev. Lett. 88, 257002 (2002).

15 J. Bardeen, L. N. Cooper, and J. R. Schrieffer, Phys. Rev. 1175, 108 (1957).

16 H. A. Jahn and E. Teller, Proc. Roy. Soc. A161, 220 (1937).

17 K. M. Shen, F. Ronning, D. H. Lu, W. S. Lee, N. J. C. Ingle, W. Meevasana, F. Baumberger, A. Damascelli, N. P. Armitage, L. L. Miller, et al., Phys. Rev. Lett. 93, 267002 (2004).

18 L. D. Landau, Sov. Phys. 3, 664 (1933).

19 M. Hengsberger, D. Purdie, P. Segovia, M. Garnier, and Y. Baer, Phys. Rev. Lett. 83, 592 (1999).

20 O. P. Sushkov, G. A. Sawatzky, R. Eder, and H. Eskes, Phys. Rev. B 56, 11769 (1997).

21 A. S. Mishchenko, N. V. Prokof'ev, and B. V. Svistunov, Phys. Rev. B 64, 033101 (2001).

22 O. Rösch and O. Gunnarsson, Phys. Rev. Lett. 92, 146403 (2004).

${ }^{23}$ L. Pintschovius, D. Reznik, W. Reichardt, Y. Endoh, H. Hiraka, J. M. Tranquada, H. Uchiyama, T. Masui, and S. Tajima, Phys. Rev. B 69, 214506 (2004).
24 J. J. M. Pothuizen, R. Eder, N. T. Hien, M. Matoba, A. A. Menovsky, and G. A. Sawatzky, Phys. Rev. Lett. 78, 717 (1997).

25 G. Martinez and P. Horsch, Phys. Rev. B 44, 317 (1991).

26 D. M. Larsen, Phys. Rev. 144, 697 (1966).

27 H. DeRaedt and A. Lagendijk, Phys. Rev. B 27, 6097 (1983).

28 P. E. Kornilovitch, Phys. Rev. Lett. 81, 5382 (1998).

29 F. C. Zhang and T. M. Rice, Phys. Rev. B 37, 3759 (1988).

${ }^{30}$ L.-C. Ku, S. A. Trugman, and J. Bonča, Phys. Rev. B 65, 174306 (2002).

31 P. B. Allen, Phys. Rev. B 3, 305 (1971).

32 D. J. Scalapino, Superconductivity (Marcel Dekker, New York, 1969).

33 S. I. Vedeneev and V. A. Stepanov, Physica C 162-164, 1131 (1989).

34 S. M. Shapiro, G. Shirane, and J. D. Axe, Phys. Rev. B 12, 4899 (1975).

35 G. D. Mahan, Many-Particle Physics (Kluwer Academic/Plenum Publishers, 2000), 3rd ed.

36 A. Macridin, G. A. Sawatzky, and M. Jarrell, Phys. Rev. B 69, 245111 (2004).

37 A. Macridin, Ph.D. thesis, University of Groningen, http://irs.ub.rug.nl/ppn/25013585X (2003).

38 N. W. Ashcroft and N. D. Mermin, Solid State Physics (Brooks Cole, 1976).

39 I. G. Lang and Y. A. Firsov, Sov. Phys. JEPT 16, 1301 (1963).

40 The breathing mode actually can be properly defined only at the zone corner in $2 \mathrm{D}$ and at $k=\pi$ in $1 \mathrm{D}$. However in this paper we extend this nomenclature for all the $k$ points, even if this is not entirely correct.

41 We set the lattice constant equal to unity.

42 DOS for the linear dispersion is constant $=1 / v_{F}$ while for a 1D tight-binding dispersion it is infinite at $k=0$.

43 The physics at energies close to $\omega_{0}$ relative to Fermi surface is presumably not influenced much by the electron hole creation at the Fermi surface. 